\title{
CORONAVIRUS
}

\section{Hydrogen peroxide mouthwash}

A commentary on this letter has been published.

Sir, there is a commercially available mouthwash containing $1.5 \%$ effective hydrogen peroxide on the market (Peroxyl, Colgate-Palmolive Ltd) which is above the recommended $0.5 \%$ concentration required to be virucidal to coronavirus. ${ }^{1}$ It may therefore be worth recommending the routine use of such a mouthwash pre-operatively when dental treatment is to be delivered as urgent care, so any subsequent droplet contamination from the oropharyngeal complex can be rendered potentially less infective.

Following a specific study on droplet contamination on surfaces by COVID-19 with various dental procedures, it may also substantially modify or reduce the decontamination time between patients and render the working environment for our professionals safer.

\section{R. Saravanamuttu, London, UK}

\section{Reference}

1. Romesh A, Thomas J T, Muraliharan N P, Vargese S S Efficacy of adjunctive use of hydrogen peroxide with chlorhexidine as a procedural mouthwash on dental aerosol. Nat J Physiology, Pharmacy Pharmacology 2015; 5: 431-435.

\section{https://doi.org/10.1038/s41415-020-1643-2}

\section{High aerosol generating potential}

Sir, the current pandemic situation has forced us to re-evaluate and adapt perhaps most parts of our practice which we (unfortunately?) took for granted it seems. Inhalation sedation (IS), possessing a high aerosol generating potential is certainly not immune to the debate. The unique challenge in this case is that IS has been constantly addled by numerous regulations on top of varied guidelines (IACSD, EAPD, ANZCA,
AAP) depending upon which region of the world one happens to be practising in. These guidelines vary over a wide spectrum of specifications such as written confirmation of informed consent (IACSD, EAPD only), pulse oximeter and documented clinical monitoring (ANZCA), training in paediatric advanced life support and IV access (AAP), formal IS training for practitioners and $\mathrm{CPR}$ training (ANZCA, IACSD). ${ }^{1}$ There are even differences in equipment installation and maintenance recommendations. ${ }^{1}$

A testament to the possible variation of regulations which would in turn affect the way IS is practised is the fact that across Europe, 51 countries possess individual regulations for IS practice and 53 separate state dental boards in the USA regulate IS practice in their areas of jurisdiction. These guidelines are then subject to the recommendations of various local regulatory bodies.

It is surprising to note that quite a few regions of the world do not follow a given set of guidelines. This glaring oversight becomes more pronounced in today's situation where practitioners are preferring extractions over routine endodontics and for cases where children might have to undergo extraction of painful/abscessed teeth. Clarity on the safety of IS would help resolve a number of dilemmas today and in the future.

It might be argued that oral sedation is used if doubts exist. While that might be valid to an extent, the predictability of IS should outweigh this as COVID-19 is not the only medical risk of which one should be wary. Surely there is a limit as to how much out of our way we can go to avoid the virus? IS should be preferred over inhalational sedation in situations wherein a choice must be made; it certainly trumps oral sedation as far as predictability and dose adjustment are concerned.

Then again, not all guidelines insist upon formal IV access training. So we can see how this is a problem. The guidelines are as varied as the challenges presented and there are more chances of encountering lacunae in practice. It seems that unifying IS practice protocols is needed now more than ever to bring clarity. This includes the roles of each team member, following routine decontamination procedures, operatory ventilation and attendance guidelines, and above all in relation to aerosol generation, use it only when absolutely necessary and when no other viable alternative presents itself. Vaibhav Sahni, New Delhi, India

\section{Reference}

1. Rajan S, Manton D J, Bhujel N. A review of contemporary inhalation sedation guidelines and regulations related to treating children. Faculty Dent J 2017; 8: 112-118.

https://doi.org/10.1038/s41415-020-1700-x

\section{Substance 'disuse' during crisis}

Sir, the fatality rate of COVID-19 with no pre-existing comorbidity is on average $0.9 \%$ while tobacco kills millions of healthy individuals worldwide. ${ }^{1}$ The failure of substance abusers to quit is explained by their addictive and euphoric action. Strict lockdown measures and supply of only essential services have dampened the sale of tobacco and its products. Socialising promotes substance abuse, whereas self-isolation and family bonding time may provide support linked to smokers attempting to quit, achieve abstinence and overcome withdrawal. ${ }^{2}$ Internet-based cognitive behaviour may minimise loneliness and the inclination towards substance abuse. ${ }^{3}$ 